This item was submitted to Loughborough's Research Repository by the author.

Items in Figshare are protected by copyright, with all rights reserved, unless otherwise indicated.

\title{
Distinguishing valid from invalid causal indicator models
}

PLEASE CITE THE PUBLISHED VERSION

http://dx.doi.org/10.1080/15366367.2016.1264235

\section{PUBLISHER}

(c) Taylor \& Francis

\section{VERSION}

AM (Accepted Manuscript)

\section{PUBLISHER STATEMENT}

This work is made available according to the conditions of the Creative Commons Attribution-NonCommercialNoDerivatives 4.0 International (CC BY-NC-ND 4.0) licence. Full details of this licence are available at: https://creativecommons.org/licenses/by-nc-nd/4.0/

\section{LICENCE}

CC BY-NC-ND 4.0

\section{REPOSITORY RECORD}

Cadogan, John W., and Nick Lee. 2019. "Distinguishing Valid from Invalid Causal Indicator Models". figshare. https://hdl.handle.net/2134/23066. 


\section{Distinguishing Valid from Invalid Causal Indicator Models}

John W. Cadogan

Professor of Marketing

School of Business and Economics

Loughborough University

UK

And

Professor of Marketing

LUT School of Business and Management

Lappeenranta University of Technology

Finland

J.W.Cadogan@Lboro.ac.uk

Nick Lee

Professor of Marketing

Warwick Business School

Warwick University

Nick.Lee@wbs.ac.uk

Under consideration in Measurement: Interdisciplinary Research \& Perspectives 


\title{
Distinguishing Valid from Invalid Causal Indicator Models
}

\begin{abstract}
We highlight the difference between valid causal indicator models, that provide useful information on the variance of theoretical latent variables, and invalid causal indicator models, which do not. We suggest that invalid causal indicator models are of the type typically used in the causal indicator literature, and urge for research to reflect on how to advance the use of valid causal indicator models.
\end{abstract}

\section{Introduction}

We applaud the paper by Aguirre-Urreta, Rönkkö and Marakas (Omission of Causal Indicators: Consequences and Implications for Measurement), since their explanations and simulations work towards demystifying causal indicator models, which are often used by scholars wishing to measure latent variables. In this comment, we focus on the utility of using causal indicator models to provide information on latent variables, reflecting on the conditions under which causal indicator models may provide valid conclusions about the variance of the latent variables they purportedly measure, and specifying the conditions under which causal indicator models should not be used in this regard. We conclude that causal indicator models (a) are often used inappropriately, and so do not provide valid information on the latent variable one wishes to model, (b) are (possibly too) unwieldy as methods of providing valid information on a focal latent variable, and (c) should probably be abandoned in favor of reflective methods for measuring constructs of interest until additional research is conducted to provide guidance on how to use them to make valid conclusions about focal latent variables. 


\section{Causal indicator models and their place in measurement}

Essentially, measurement is about quantifying the properties of objects, and researchers often measure properties of objects in order to test theories about the nature of the causal forces in the world. For instance, a researcher may speculate that variance in $\eta 1$, some real latent variable (see Cadogan et al. 2013), has some causal impact on other variables. In order for the researcher to test their ideas, they must have information on how $\eta 1$ varies, together with information on its covariance with the other variables in the nomological network. The problem here is that $\eta 1$ is latent, and so data on its variance (and covariance) is not directly observable - it is missing. Accordingly, researchers are interested in finding ways to infer the variance of $\eta 1$ using data that can be directly observed.

One option is to seek data from trace evidence that a latent variable has varied (see Figure 1): here, the researcher believes that some closely related outcome of $\eta 1, Y 1$, can perform this job. Thus, variance in $\eta 1$ is assumed to cause variance in Y1, and so one can make inferences about $\eta 1$ 's variance using directly observed data about Y1's variance, and by making some assumptions about the magnitude of the relationships between $\eta 1$ and $\mathrm{Y} 1(\lambda 1)$, and the relationship between a unique factor (u1) and $\mathrm{Y} 1(\Theta 1)$. This approach is commonly called reflective measurement. $^{1}$

A second possible method for generating information on the variance of a latent variable could be to seek data on variables that are known to cause variance in the latent variable. This is the causal indicator model, and Figure 2 demonstrates it. Here, the Xs are the entire set of variables that cause variance in $\eta 1$. If one wants to construct the variance of $\eta 1$ using the Xs, then, ideally one would (a) know the identity of every $X$ in the entire set of $X$ variables that cause $\eta 1$, (b) know the form 
and magnitude (the $y s$ ) of the relationships between each of the Xs and $\eta 1$, and (c) have directly observed data on the variances and covariances of each X. Under these conditions, one could consider the Xs to be the set of causal indicators of $\eta 1$.

Figure 1: Reflective measurement of $\eta 1$

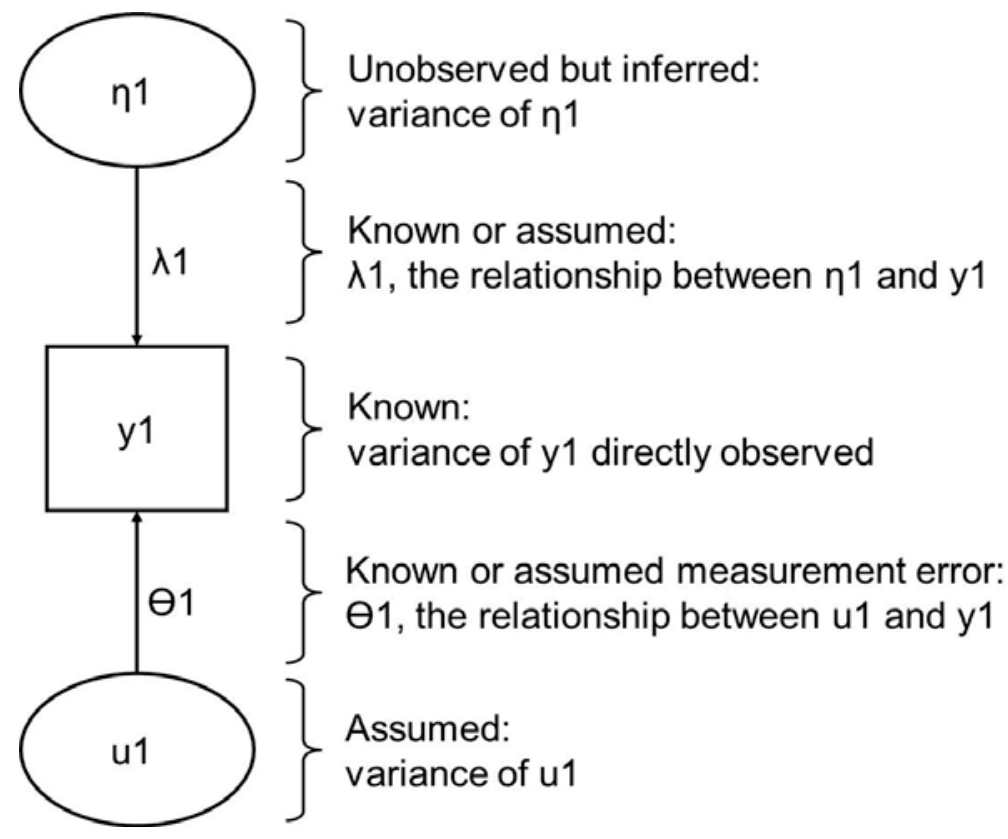

Figure 2: Causal indicator model of $\eta 1$

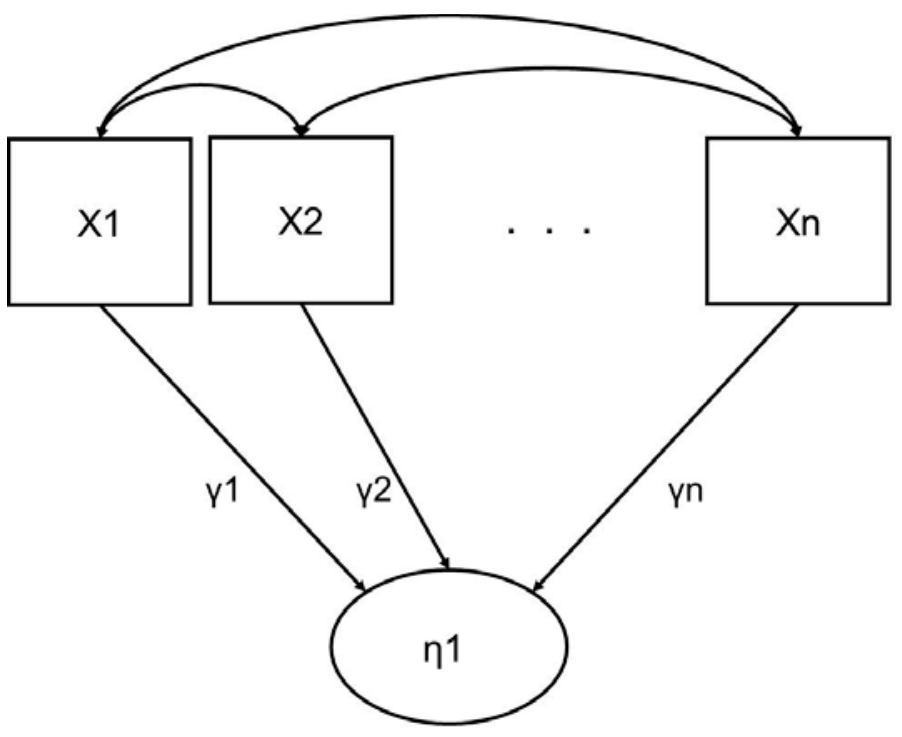

Known:

variances and covariances of Xs are directly observed

Known:

the form and the magnitude (the ys) of the relationships between the $X s$ and $\eta 1$

Unobserved but inferred: variance of $\eta 1$ 
Of course, the ideal model has limitations, with perhaps the most obvious being that the researcher may not know the identity of every causal indicator in the entire set of variables that cause $\eta 1$. Figure 3 demonstrates the revised causal indicator model under this latter condition.

Figure 3: Causal indicator model of $\eta 1$ with some unknown causal indicators

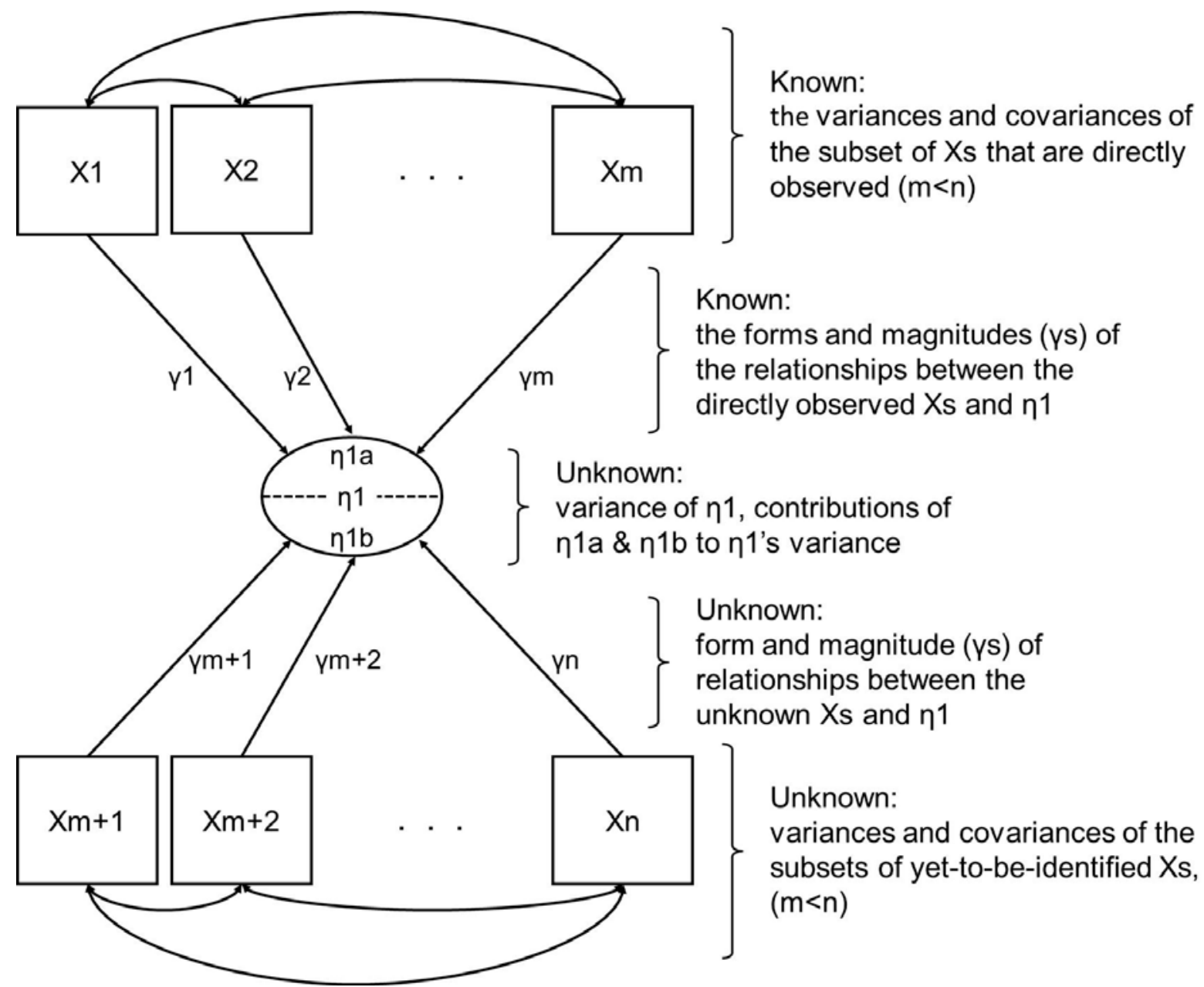

Here, data from the known Xs can be used (together with the information on the form and magnitude of their relationships with $\eta 1$ ) to create information on a fraction of $\eta 1$ 's variance, labeled $\eta 1 \mathrm{a}$ in Figure 3. Unfortunately, here, we are now faced with a situation where it is impossible to make any inference about $\eta 1$ 's 
variance because there is another fraction of $\eta 1$ 's variance, $\eta 1 \mathrm{~b}$ in Figure 3 , that we have no information on, and neither do we know the relative contributions of $\eta 1 \mathrm{a}$ and $\eta 1 \mathrm{~b}$ to $\eta 1$ 's variance.

However, if one were comfortable estimating the relative contributions of $\eta 1 \mathrm{a}$ and $\eta 1 \mathrm{~b}$ to $\eta 1$ 's variance, one could potentially model $\eta 1$ as shown in Figure 4 . Here, the missing Xs are summarized using the proxy variable $z 1$, and the contribution of $\mathrm{z} 1$ to $\eta 1$ is specified. Models of this kind can be estimated, and $\eta 1$ 's variance can be modelled.

Figure 4: Causal indicator model of $\eta 1$ where unknown causal indicators' variance contribution is specified

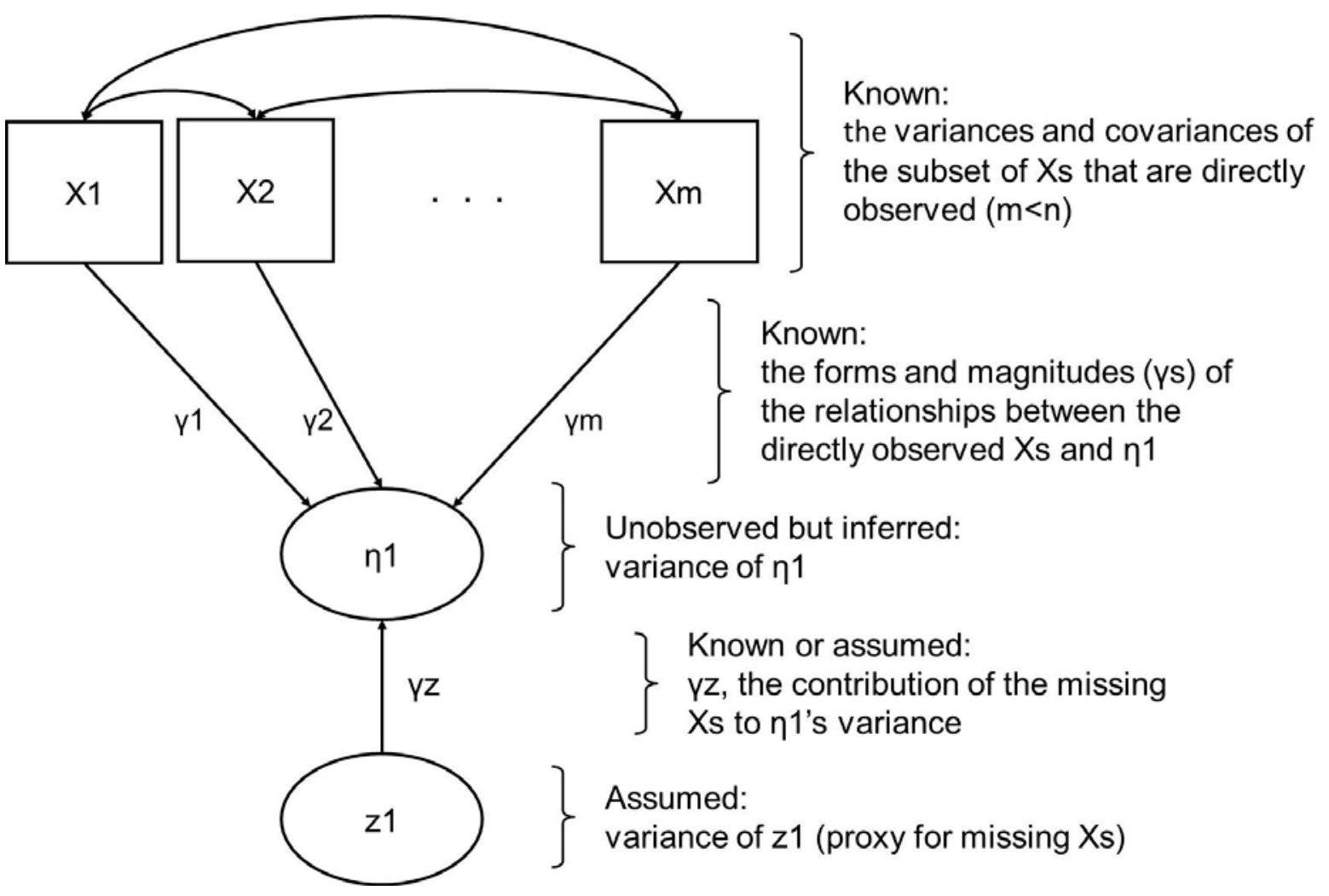




\section{Invalid causal indicator models}

So far, we have discussed causal indicator models in which the focal latent variable, $\eta 1$, is predicted using its causes, and where the correspondence between the variance of the predicted variable and the variance of the real world $\eta 1$ is considered to be fundamental. Indeed, unless the measured causal indicators' contribution to $\eta 1$ 's variance is known and specified, then the ability to say anything at all about $\eta 1$ 's variance is severely compromised. However, our approach to causal indicators is not consistent with most of the literature on causal indicators. Why? Because in the traditional causal indicator model, key features of the model are specified as unknown. Specifically, in the traditional causal indicator model, researchers believe that they are modelling $\eta 1$ as shown in Figure 5.

Figure 5: Typical implementation of causal indicator model of $\eta 1$

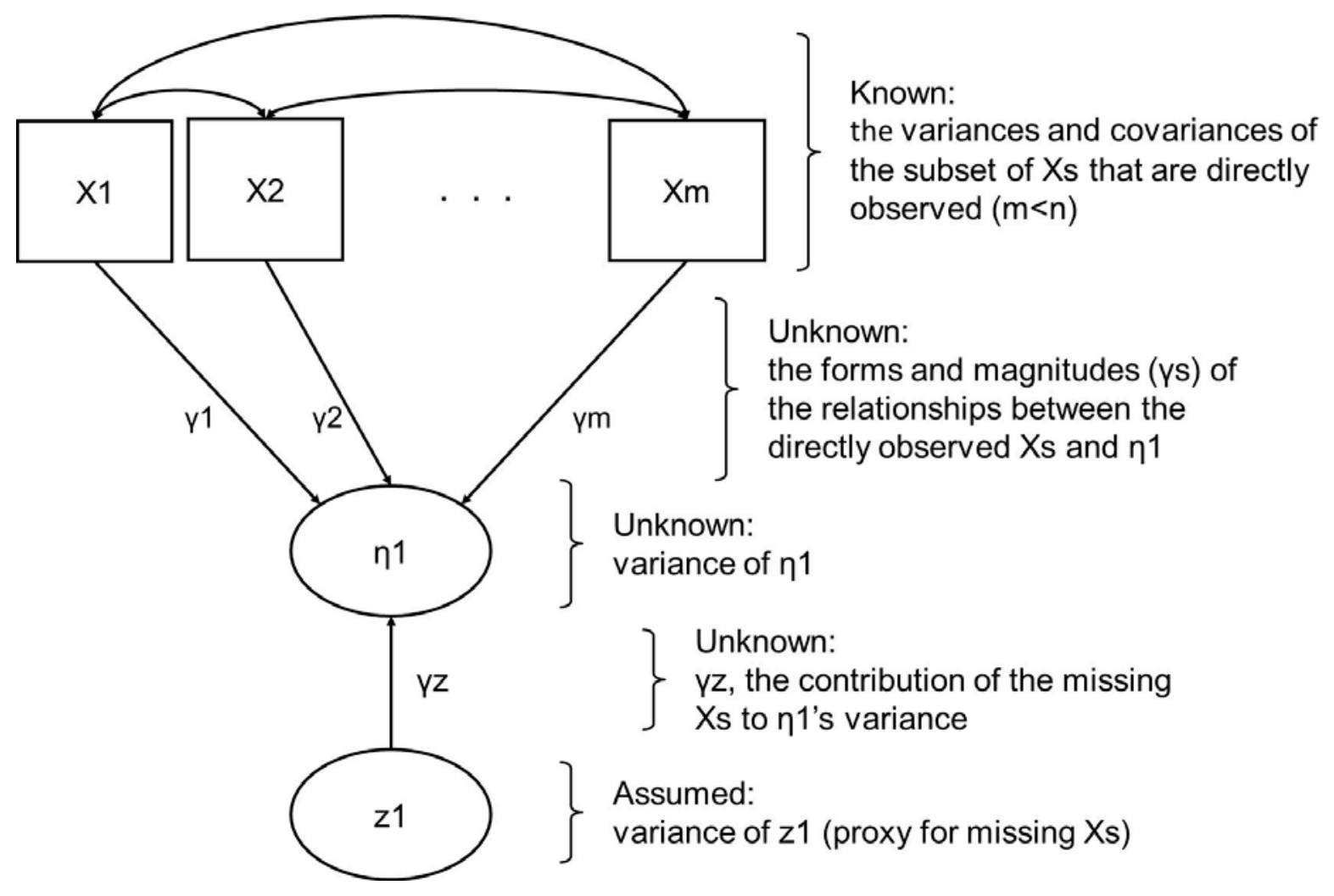


In Figure 5, there is simply not enough information provided to ever be able to say anything about $\eta 1$ 's variance. Yet researchers persist in using it, under the impression that the Figure 5 model is a useful way of modeling variance in $\eta 1 . A$ typical way that they do this is to (unwittingly) create $\eta 1$ 's variance using information that is entirely downstream of $\eta 1$ in a causal model. Indeed, this is the finding that Aguirre-Urreta et al. report in their paper. It is no surprise, then, that the authors demonstrate that the traditional version of the causal indicator model is not feasible as way of getting information on $\eta 1$.

Indeed, the traditional causal indicator model, containing downstream latent variables to help with identification or to "test" theory about the focal latent variable's causal outcomes, as shown in Figure 6, is an invalid way of modeling $\eta 1$ 's variance, since the focal latent variable (here depicted as $\eta 2$ ) receives its empirical meaning from its downstream variables ( $\eta 3, \eta 4$ and $\eta 5)$, and yet the shared variance components of the downstream variables may not correspond to the true variance of $\eta 1$. Indeed, to emphasize this point, in Figure 6 it can be seen that there is no $\eta 1$, since nowhere in the model is $\eta 1$ 's variance explicitly predicted or estimated.

\section{Where to next?}

We wholeheartedly agree with Aguirre-Urreta et al.'s inference, then, that the use of traditional causal indicator models should be reconsidered. However, we also believe that there is scope for additional research into the use of "true" causal indicator models. True causal indicator models are of the kind shown in Figure 2 and Figure 4 , in which variance in the focal latent variable, $\eta 1$, is predicted by its causes, and where the correspondence between the variance of the predicted variable and 
the variance of the real world $\eta 1$ is an explicit feature of the model. In reality, Figure 2 may be unlikely in practice, since the following potential unknowns are possible:

Figure 6: Typical implementation of causal indicator model of $\eta 1$ with downstream variables for identification

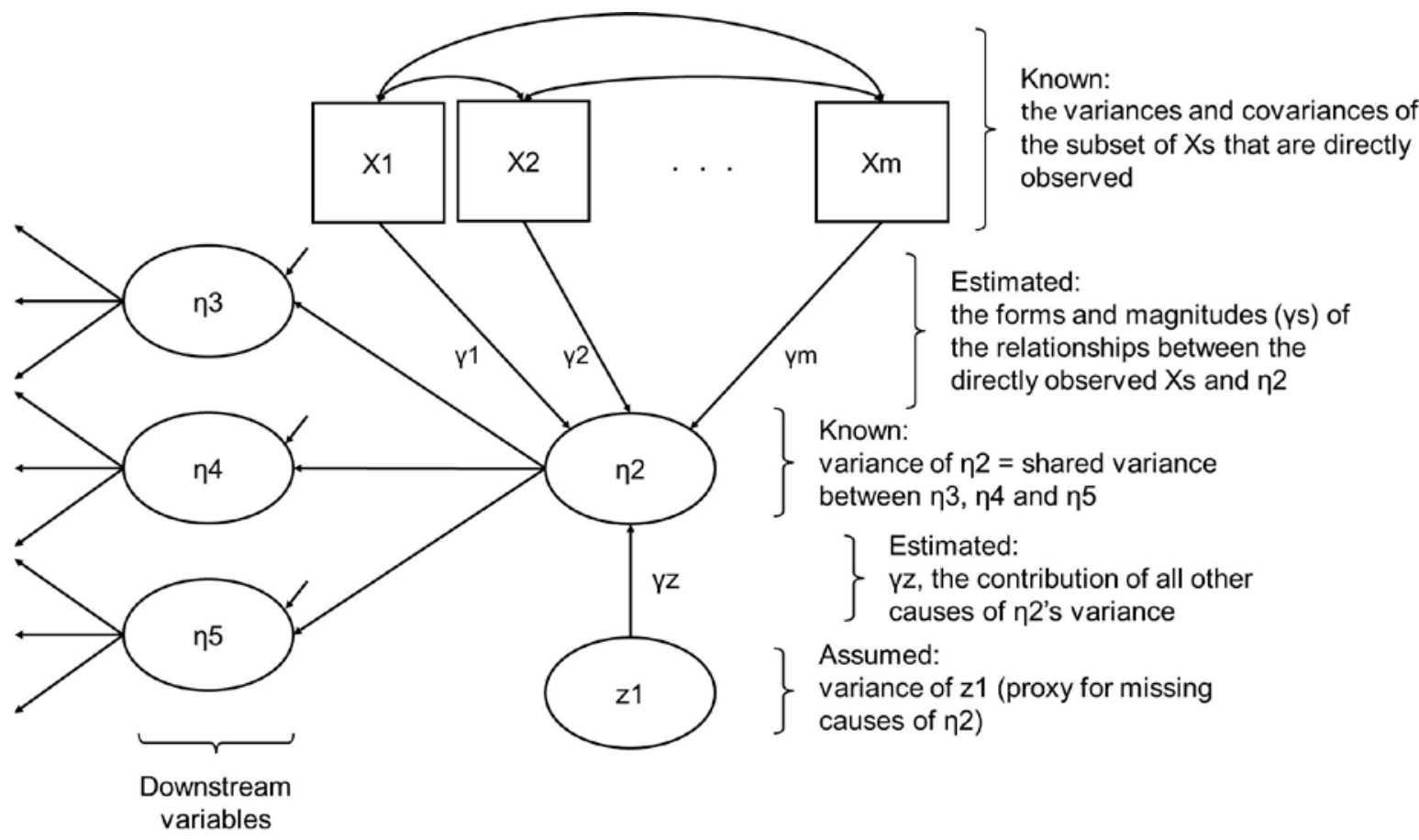

(a) some of the $X$ variables may be unknown,

(b) the forms of some of the relationships between the Xs and $\eta 1$ may be unknown,

(c) the magnitudes of some of the relationships between the Xs and $\eta 1$ may be unknown (even if the forms are known),

(d) the measurements of the Xs may be imperfect, and

(e) the contribution to $\eta 1$ 's variance coming from the known Xs, and the contribution to $\eta 1$ 's variance coming from the unknown Xs, may be unknown. 
Thus, in order to run a true causal indicator model, it may be necessary to guess or otherwise impute some of these unknown values. Research is therefore needed in order to identify best practices on this front. For instance, research could focus on ascertaining the effects of "error in prediction" on model testing, where the less the contribution to $\eta 1$ 's variance coming from the measured $X s$, the greater the error in prediction. Until such time as research has built an understanding of the potential for invalid / valid model testing using the true causal indicator model, we suggest that measurement should progress using reflective measurement models. Indeed, given that true causal indicator models may contain very large numbers of causal indicators, and require large amounts of a priori knowledge about relationships between indicators and focal latent variables, the complexity of the true causal indicator model may simply make it too unwieldy for most measurement purposes.

\section{Endnotes}

1. In Figure 1, for simplicity, we present a reflectively measured latent variable that only has one piece of trace evidence, Y1. Reflective measures containing multiple indicators are also possible.

\section{References}

Aguirre-Urreta, Miguel I., Mikko Rönkkö and George M. Marakas (forthcoming), "Omission of Causal Indicators: Consequences and Implications for Measurement," Measurement: Interdisciplinary Research \& Perspectives.

Cadogan, John W., Nick Lee and Laura Chamberlain (2013), "Formative Variables are Unreal Variables: Why the Formative MIMIC Model is Invalid," AMS Review, Vol. 3, No. 1, pp. 38-49. 